14. Lange KW, Paul GM, Naumann M, Gsell W. Dopaminergic effects on cognitive performance in patients with Parkinson's disease. J Neural Transm 1995;46(suppl):423-432.

15. Becker G, Bogdahn U, Straßburg H-M, et al. Identification of ventricular enlargement and estimation of ventricular pressure by transcranial color-coded real-time sonography. J Neuroimaging 1994;4:17-22.

16. Berg D, Grote C, Rausch WD, et al. Iron accumulation in the substantia nigra in rats visualized by ultrasound. Ultrasound Med Biol 1999;25:901-904.

17. Youdim MBH, Ben-Shachar D, Eshel G, Finberg JPM, Riederer
P. The neurotoxicity of iron and nitric oxide. Relevance to the etiology of Parkinson's disease. Adv Neurol 1993;60:259-266.

18. Dexter DT, Sian J, Jenner P, Marsden CD. Implications of alterations in trace element levels in brain in Parkinson's disease and other neurological disorders affecting the basal ganglia. Adv Neurol 1993;60:273-281.

19. Gerlach M, Ben-Shachar D, Riederer P, Youdim MBH. Altered brain metabolism of iron as a cause of neurodegenerative diseases? J Neurochem 1994;63:793-807.

20. Jellinger KA. The role of iron in neurodegeneration. Drugs Aging 1999;14:115-160.

\title{
cME Competency to consent to medical treatment in cognitively impaired patients with Parkinson's disease
}

\author{
Maureen P. Dymek, PhD; Paul Atchison, MD; Lindy Harrell, MD, PhD; and Daniel C. Marson, JD, PhD
}

Article abstract-Objectives: To investigate capacity to consent to medical treatment (competency) in cognitively impaired patients with PD. Background: Although competency has been studied empirically in patients with cortical dementia (AD), no empirical studies have examined competency in patients with PD or other subcortical neurodegenerative disorders. Methods: Patients with PD with cognitive impairment $(\mathrm{n}=20)$ and older controls $(\mathrm{n}=20)$ were compared using a standardized competency measure (Capacity to Consent to Treatment Instrument [CCTI]) and neuropsychological test measures. The CCTI tests competency performance and assigns outcomes (capable, marginally capable, incapable) under four different legal standards (LS). Results: Patients with PD performed below controls on the four LS: capacity to evidence a treatment choice (LS1) $(p<0.03)$, capacity to appreciate consequences of a treatment choice (LS3) $(p<0.03)$, capacity to provide rational reasons for a treatment choice (LS4) $(p<0.0001)$, and capacity to understand the treatment situation and choices (LS5) $(p<0.0001)$. With respect to competency outcomes, patients with PD demonstrated increasing compromise (marginally capable or incapable outcomes) across the four standards: LS1 (25\%), LS3 (45\%), LS4 (55\%), and LS5 (80\%). In the PD group, simple measures of executive function (the Executive Interview) and to a lesser extent memory/orientation (Dementia Rating Scale, Memory subscale) were key predictors of competency performance and outcome on the LS. Conclusions: Cognitively impaired patients with PD are likely to have impaired consent capacity, and are at risk of losing competency over the course of their neurodegenerative illness. Patients with PD have particular difficulty meeting more stringent, clinically relevant competency standards that tap reasoning skills and comprehension of treatment information. Executive dysfunction appears to be a primary neurocognitive mechanism for competency loss in PD.

NEUROLOGY 2001;56:17-24

Between one quarter and one half of patients with $\mathrm{PD}$ are estimated to also have dementia..$^{1-3}$ Cognitive deficits in PD commonly emerge in the areas of higher order attention, memory, spatial skills, and in particular, executive function. ${ }^{4,5}$ Executive function

Additional material related to this article can be found on the Neurology Web site. Go to www.neurology.org and scroll down the Table of Contents for the January 9 issue to find the title link for this article. deficits are among the most prominent cognitive deficits in PD, and may be one of the earliest signs of cognitive deterioration in the disorder.4,6,7 Furthermore, executive function deficits may underlie other cognitive impairments in PD, such as memory, verbal fluency, reasoning, spatial skills, and complex attention..$^{4,5}$

Cognitive deficits presumably contribute to functional declines experienced by patients with $\mathrm{PD}$.

From the Department of Psychiatry (Dr. Dymek), University of Chicago, IL; Department of Neurology (Drs. Atchison, Harrell, and Marson), Alzheimer's Disease Research Center (Drs. Harrell and Marson), and Center for Aging (Drs. Harrell and Marson), University of Alabama at Birmingham; and the Veterans' Administration Medical Center (Dr. Harrell), Birmingham, AL.

Supported in part by an Alzheimer's Disease Center Cores grant (NIH, NIA 1 P30 AG10163-1), an Alzheimer's Disease Program Project grant (NIH, NIA 5 P01 AG06569-05), an Alzheimer's Disease Research Center grant (NIH, NIA 1P50 AG16582-01), and an Alzheimer's Association Pilot Research Grant (PRG 91-122).

Received April 24, 2000. Accepted in final form September 28, 2000.

Address correspondence and reprint requests to Dr. Daniel Marson, Department of Neurology, JT1216, University of Alabama at Birmingham, Birmingham, AL; e-mail: dmarson@uab.edu 
However, little is known about the relationship of neurocognitive change to declines in patients' higher order, independent activities of daily life (IADL), such as medical decision making. The capacity to consent to medical treatment (competency) is important to patients with PD and their families, who must make complex decisions about medical care over the course of a protracted and progressive neurodegenerative disease. In addition, consent capacity is an integral element of informed consent to treatment, ${ }^{8,9}$ which requires that a patient's consent be voluntary, informed, and competent. ${ }^{9,10}$ Without a proper informed consent, medical treatment provided to a patient is legally a technical battery, even if the treatment is benign and intended to benefit the patient. ${ }^{11}$ Thus, potential impairments in the consent capacity of patients with PD have important medical, legal, and ethical implications for neurologists and other health-care providers.

Patients with both mild and moderate $\mathrm{AD}$ show significant deficits in their capacity to consent to medical treatment. ${ }^{12,13}$ However, no studies to date have examined competency in patients with $\mathrm{PD}$ or other subcortical neurodegenerative disorders. Competency loss in $\mathrm{PD}$ may differ from that found in $\mathrm{AD}$, given the different neurologic basis and neuropsychological profiles of these disorders. The current study investigates the capacity of cognitively impaired patients with PD to consent to medical treatment, and also explores the relationship of neurocognitive change to competency loss in the PD group.

Methods. Participants. Twenty patients, aged 60 years and older with idiopathic PD and related cognitive impairment, were recruited from a movement disorders clinic. After conducting chart reviews to determine eligibility, selected patients with PD were provided handouts describing the study, and follow-up phone calls were made to interested patients. Inclusion criteria consisted of the following: 1) a positive diagnosis of idiopathic PD by a movement disorders neurologist (P.A.) based on symptoms, examination, disease course, and response to antiparkinsonian medication; 2) patient- or caregiver-reported complaints of cognitive impairment; and 3) a Mattis Dementia Rating Scale $^{14}$ (DRS) total score of 130 and below. Exclusion criteria consisted of the following: 1) positive history of major neurologic conditions other than PD; 2) a positive history of major psychiatric disorders (including major depression but excluding minor depression and dysthymia); 3) current or past use of neuroleptics; 4) a concomitant medical illness adversely affecting cognition, e.g., obstructive pulmonary disease; 5) a history of substance abuse; and 6) prior neurosurgical intervention. All patients with PD were taking antiparkinsonian medications. The mean age of the $\mathrm{PD}$ group was 75 years $(\mathrm{SD}=7.5$ years), and the mean education of the group was 14.3 years $(\mathrm{SD}=$ 7.5 years). The mean DRS total score for the PD group was 117.3 ( $\mathrm{SD}=14.5)$, reflecting an overall mild level of cognitive impairment. Older control subjects consisted of 20 healthy, community-dwelling adults aged 60 years and older without significant medical or mental illness (including depression). Controls were previously recruited from a Program Project in AD (NIA 5P01 AG06569-05) and an AD Center Core (NIA 1P30 AG101163-1). Control subjects were screened using neurologic, psychiatric, neuropsychological, neuroradiologic (including cranial MRI or CT scanning), and laboratory evaluations. Assignment as a control status was made by consensus of a neurologist, psychiatrist, and neuropsychologist. The mean age of the control group was 68.1 years ( $\mathrm{SD}=7.9$ years), and the mean duration of education was 14.8 years ( $\mathrm{SD}=2.3$ years). The mean DRS total score for the group was $141.2(\mathrm{SD}=3.1)$, which is indicative of normal cognitive function.

Measures. Competency measure. Capacity to consent to medical treatment was measured with the Capacity to Consent to Treatment Instrument (CCTI). ${ }^{12,15,16}$ The CCTI is a reliable and valid instrument for the assessment of consent capacity in older control subjects and patients with mild and moderate $\mathrm{AD} .{ }^{12,15}$ The CCTI employs two specialized clinical vignettes that each present a hypothetical medical problem (neoplasm, atherosclerotic heart disease) and symptoms, and two treatment options with associated risks and benefits. The administration format for each vignette approximates an informed consent dialogue, and requires the subject to process oral and written information concerning the medical problem, symptoms, treatment options, and risks/benefits. Subjects then answer questions designed to test consent capacity under each of four discrete, established standards or thresholds drawn from legal case law and psychiatric literature. ${ }^{17,18}$ These standards, as operationalized by the current authors, are presented below in order of increasing difficulty for dementia patients ${ }^{12}$ :

LS1: Simply evidencing/communicating a treatment choice

LS3: Appreciating the practical and anticipated consequences of a treatment choice

LS4: Providing rational reasons (pro and con) for a treatment choice

LS5: Understanding the treatment situation, and treatment choices, and respective risks/benefits

In addition to these four standards, we also test for a consent related ability described as making the "reasonable" treatment choice (when the alternative is unreasonable), ${ }^{17,19}$ which we reference as [LS2]. [LS2] is not an accepted legal standard for judging consent capacity, because of concerns about arbitrariness in determining what constitutes a "reasonable choice.". ${ }^{19}$ Accordingly, [LS2] is referenced in brackets to distinguish it from the other four established LS. However, we continue to use [LS2] in order to better understand treatment preferences of neurologic patients..$^{12,20}$

CCTI administration procedures. Vignettes A and B of the CCTI were presented orally and simultaneously in writing to all subjects, in an uninterrupted disclosure format. ${ }^{21}$ The vignettes were administered in counterbalanced order to control for possible order effects. All subject responses were audiotaped and transcribed to ensure accurate scoring. CCTI protocols were scored by trained staff according to a detailed protocol. ${ }^{12}$ 
Table 1 Group comparisons on demographic, dementia screen, and legal standard (LS) variables

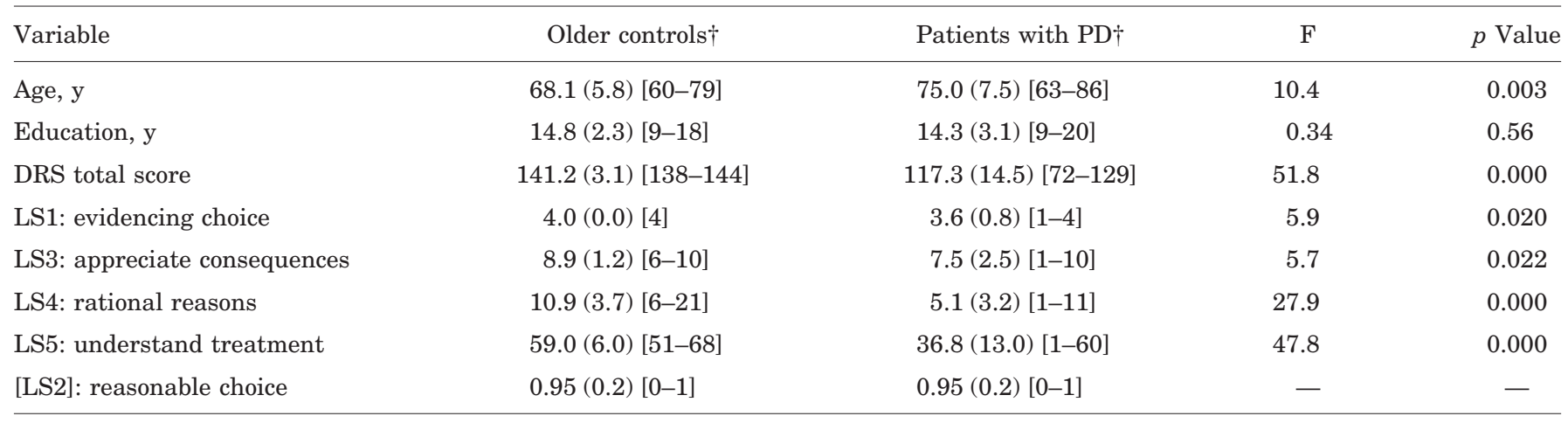

Values expressed as mean (SD) [range].

DRS $=$ Dementia Rating Scale.

Neuropsychological battery. Neurocognitive functioning was measured with a comprehensive neuropsychological battery designed to evaluate cognitive functions clinically and theoretically linked to competency and to dementia. ${ }^{12,22,23}$ Specifically, the neuropsychological battery comprised the following cognitive domains and tests:

Orientation. Orientation was measured using information and orientation items of the Wechsler Memory ScaleRevised (WMS-R). ${ }^{24}$

Attention/concentration. Attention and concentration were measured using the Mental Control and Digit Span subtests of the WMS-R, ${ }^{24}$ and the Attention subscale of the DRS. ${ }^{14}$

Expressive language. Confrontation naming was measured using the Boston Naming Test (BNT). ${ }^{25}$

Receptive language. Auditory language comprehension was measured using a Simple Auditory Comprehension test (SAC). ${ }^{26}$ Propositional auditory comprehension was measured using a short form of the Token Test. ${ }^{27}$

Visuospatial skills. Spatial construction skills were measured with the Construction subscale of the DRS. ${ }^{14}$

Memory. Short-term verbal memory was measured using the Logical Memory I subtest of the WMS-R, ${ }^{24}$ and the Memory subscale of the DRS. ${ }^{14}$ Delayed verbal recall was measured using the Logical Memory II subtest of the WMS-R. ${ }^{24}$

Executive function. Simple executive abilities were measured using the Executive Interview (EXIT-25), ${ }^{28}$ and the Initiation/Perseveration subscale of the DRS. ${ }^{14}$ Simple visuomotor planning and tracking was assessed using Trails A of the Halstead-Reitan battery. ${ }^{29}$ Visuospatial tracking, planning, and mental flexibility was measured using Trails B. ${ }^{29}$ Phonemic and semantic word fluency were measured using Controlled Oral Word Fluency $(\mathrm{COWF})^{30}$ and Animal Naming. ${ }^{31}$

Verbal conceptualization and reasoning. Verbal conceptualization and reasoning were measured using the Similarities subtest of the Wechsler Adult Intelligence ScaleRevised (WAIS-R), ${ }^{32}$ and the Conceptualization subtest of the DRS. ${ }^{14}$

Judgment. Judgment was measured using the Comprehension subtest of the WAIS-R. ${ }^{32}$
Mood. Mood was assessed with the Geriatric Depression Scale (GDS). ${ }^{33}$

Data analyses. Demographic variables. The PD and control groups were compared on demographic variables using analysis of variance (ANOVA). All data analyses were conducted using SPSS version 6.0 (Chicago, IL), ${ }^{34}$ unless otherwise noted.

Competency performance on LS. For each LS, subject scores were summed across the two vignettes to create a composite LS variable (except for [LS2], which is specific to vignette $\mathrm{A})$. $\mathrm{PD}$ and control groups were compared on these composite LS variables using ANOVA (LS 1, 3 to 5). Because [LS2] was scored on a dichotomous basis (pass/ fail), $\chi^{2}$ analysis was used for this variable.

Competency outcomes on LS. Because competency is ultimately a categorical status, we were interested in the competency outcome status (capable, marginally capable, incapable) of subjects with PD for each LS. As discussed below, these outcomes were identified psychometrically for scientific purposes and do not purport to represent the actual legal competency status of the participants. For each of the three LS using interval data (LS3 to 5), the performance of a patient with $\mathrm{PD}$ was classified into one of the three competency outcomes using cut off scores statistically derived from control performance. Cut off scores were established that defined capable as a score equal to or greater than $1 \frac{1}{2} \mathrm{SD}$ below the control mean on the LS, marginally capable as a score falling between $1 \frac{1}{2}$ and $2 \frac{1}{2}$ SD below the control group mean on the LS, and incapable as a score falling below $2 \frac{1}{2} \mathrm{SD}$ below the control mean on the LS. Although statistical approaches to assigning competency status inevitably have an arbitrary aspect, our approach reflects contemporary methods for defining neuropsychological impairment, and thus represents a theoretically based modification of the narrower cut-score approach used in our earlier competency research. ${ }^{12,23} \mathrm{~A}$ different approach was used for the two LS using categorical data (LS1 and LS2). For LS1, which has a possible score range of 0 to 4 , the cutoff for capable was set using a perfect score (4 points), with marginally capable as the next lowest possible score (3 points), and incapable as the remaining score range ( 0 to 2 points). For [LS2], which has 
Table 2 Competency outcomes by legal standard (LS) and group

\begin{tabular}{lccc}
\hline LS/group & Capable & $\begin{array}{c}\text { Marginally } \\
\text { capable }\end{array}$ & Incapable \\
\hline LS1 & $20(100)$ & $0(0)$ & $0(0)$ \\
Control & $14(70)$ & $5(25)$ & $1(5)$ \\
PD & & $3(15)$ & $0(0)$ \\
LS3 & $17(85)$ & $5(25)$ & $4(20)$ \\
Control & $11(55)$ & $0(0)$ & $0(0)$ \\
PD & & $10(50)$ & $1(5)$ \\
LS4 & $20(100)$ & & $0(0)$ \\
Control & $9(45)$ & $0(0)$ & $13(65)$ \\
PD & & $3(15)$ & $1(5)$ \\
LS5 & $20(100)$ & - & $1(5)$ \\
Control & $4(20)$ & - & \\
PD & $19(95)$ & & \\
[LS2] & $19(95)$ & & \\
Control & PD & &
\end{tabular}

Values expressed as n (\%).

Capable: For LS3-LS5, scores falling at or above 11/2 SD below the control group mean on the LS. For LS1, a score of 4 . For [LS2], a score of 1. Marginally capable: For LS3-LS5, scores falling between $1 \frac{1 / 2}{2}$ and $2 \frac{1}{2}$ SD below the control group mean on the LS. For LS1, a score of 3. No marginally competent outcomes are possible on [LS2]. Incapable: For LS3-LS5, scores falling below $2 \frac{1}{2}$ or more SD below the control group mean on the LS. For LS1, a score of $0-2$. For [LS2], a score of 0 .

a range of 0 to 1 , there were only two possible outcomes: capable ( 1 point) and incapable ( 0 points).

Neuropsychological predictors of competency performance. Univariate and multivariate analyses were conducted to identify key neurocognitive predictors of LS competency performance for the PD group. Univariate correlations were used as a data reduction step to identify potential predictors of LS performance for the multivariate analyses. For each LS, the four neurocognitive variables with the strongest univariate correlations were selected for follow-up multivariate analyses. This number of predictor variables reflected the minimum 5:1 subject to predictor ratio suggested by Tabachnick and Fidell ${ }^{35}$ for multiple regression equations. Each set of univariate predictors was entered into stepwise regression analyses for its respective LS, using LS score as the dependent variable and the selected neuropsychological measures as the predictor variables.

Neuropsychological predictors of competency outcomes. For each LS, nonparametric discriminant function analyses (DFA) using SAS NEIGHBOR ${ }^{36}$ (SAS Institute, Cary, $\mathrm{NC}$ ) were conducted to identify predictors of competency status (capable, marginally capable, and incapable) for the PD sample. As in a prior study, ${ }^{12}$ nonparametric DFA were used because of the disparity in competency status cell sizes across groups. Two DFA were conducted for each LS. The first DFA was run using the one neuropsychological variable with the highest univariate correlation as the predictor of competency status. The second DFA was run using the two neuropsychological variables with the highest univariate correlations as the predictors of competency status.

Results. Group comparisons on demographic, dementia screen, and CCTI LS variables. Table 1 compares control and PD groups on demographic, dementia screen, and CCTI LS variables. The groups did not differ in years of education ( $p=0.56$ ). However, the PD group was older (by 6.9 years) than the control group $(p<0.002)$. Follow-up analyses indicated that age did not correlate $(p<0.05)$ with any LS or neuropsychological variables. Thus the age difference was not viewed to be clinically significant. As expected, the control group scored significantly higher than patients with PD on DRS total score $(p<0.0001)$, again indicative of cognitive impairment in the PD group.

Table 1 also compares control and PD groups on LS variables. Controls performed significantly better than PD patients on $\operatorname{LS} 1[\mathrm{~F}(1,39)=5.9, p<0.02]$, LS3 $[\mathrm{F}(1,39)=5.7$, $p<0.02]$, LS4 $[\mathrm{F}(1,39)=27.9, p<0.00]$, and LS5 [F(1,39) $=$ $47.8, p<0.00]$. No group differences emerged on LS2.

Group comparisons on neuropsychological variables. As expected, patients with PD performed below controls on virtually all neurocognitive measures $(p<0.001)$. Patients with PD performed well within normal limits (mean $=3.5$, $\mathrm{SD}=2.0$ ) on a self-reported measure of depression (the GDS) $^{33}$ (for additional information related to this article, please visit the Neurology Web site at www.neurology.org and scroll down the Table of Contents for the January 9 issue to find the title link for this article). Moreover, the GDS did not emerge as a predictor of competency performance or status (see below), suggesting that in the PD group, depression was not a key factor influencing competency or neuropsychological performance.

Competency status on LS by group. Table 2 presents competency outcome results by LS and group. Patients with PD demonstrated a pattern of competency compromise (marginally capable and incapable outcomes) that related to stringency of LS: LS1 (25\%), LS3 (45\%), LS4 (55\%), and LS5 (80\%). As expected and with few exceptions, control subjects were assigned capable outcomes under the four LS and also [LS2].

Neuropsychological predictors of competency performance and outcome in the PD group. LS1 (evidencing a treatment choice). Table 3 presents the four strongest univariate correlates and also the multivariate predictors of PD patient performance on LS1. DRS Memory emerged as the only stepwise regression predictor of LS1 scores. DRS Memory correctly classified 70\% of PD patient outcomes on LS1, which was well above chance levels (33\%). Using both DRS Memory and WAIS-R Comprehension as predictor variables, a classification rate of $100 \%$ was obtained (for additional information related to this article, please visit the Neurology Web site at www.neurology.org and scroll down the Table of Contents for the January 9 issue to find the title link for this article).

LS3 (appreciating the consequences of choice) and [LS2] (reasonable choice). No neuropsychological measures correlated significantly $(p<0.05)$ with LS3 or [LS2] on the univariate level. Accordingly, LS3 and [LS2] were excluded from subsequent multivariate analyses. 
Table 3 Neuropsychological predictors of legal standard (LS) performance for patients with PD $(n=20)$

\begin{tabular}{|c|c|c|c|c|}
\hline \multirow[b]{2}{*}{ LS/predictor variable(s) } & \multicolumn{2}{|c|}{ Univariate correlation } & \multicolumn{2}{|c|}{ Stepwise regression } \\
\hline & $r$ & $p$ Value & $R^{2}$ cum & $p$ Value \\
\hline DRS Memory & 0.73 & 0.000 & 0.55 & $0.000($ Step 1) \\
\hline WAIS-R Comprehension & 0.67 & 0.002 & & \\
\hline \multicolumn{5}{|l|}{ LS4: rational reasons } \\
\hline EXIT 25 & -0.67 & 0.002 & 0.45 & $0.002($ Step 1) \\
\hline Trails B & -0.60 & 0.005 & & \\
\hline DRS Attention & 0.58 & 0.008 & & \\
\hline DRS Memory & 0.71 & 0.000 & 0.68 & $0.000($ Step 2) \\
\hline WAIS-R Comprehension & 0.70 & 0.001 & & \\
\hline WMS-R LM II & 0.65 & 0.002 & & \\
\hline
\end{tabular}

DRS = Dementia Rating Scale; WAIS-R = Wechsler Adult Intelligence Scale-Revised; EXIT 25 = the Executive Interview; WMS-LM II $=$ Wechsler Memory Scale Logical Memory II.

LS4 (rational reasons for choice). Table 3 presents univariate correlates and multivariate predictors of $\mathrm{PD}$ patient performance on LS4. EXIT 25 emerged as the only stepwise regression predictor of LS4 scores. Regarding competency status, the correct classification rate using EXIT 25 as the sole predictor of competency outcome was $90 \%$. A classification rate of $95 \%$ was obtained when EXIT 25 and Trails B were the predictor variables (for additional information related to this article, please visit the Neurology Web site at www.neurology.org and scroll down the Table of Contents for the January 9 issue to find the title link for this article).

LS5 (understanding treatment situation/choices). Table 3 presents univariate correlates and multivariate predictors of PD patient performance on LS5. EXIT 25 emerged as the Step 1 predictor and DRS Memory emerged as the Step 2 predictor of LS5 scores. Regarding competency status, the classification rate using EXIT 25 as the sole predictor variable was $90 \%$. The classification rate using both EXIT 25 and DRS Memory as predictor variables was $95 \%$ (for additional information related to this article, please visit the Neurology Web site at www.neurology.org and scroll down the Table of Contents for the January 9 issue to find the title link for this article).

Discussion. Consenting to treatment is an act of complex decision making with important medical, legal, and ethical consequences. ${ }^{37}$ Our findings suggest that, depending on the standard applied, many cognitively compromised patients with PD may be impaired or even lack capacity to consent to treatment. Our findings also suggest that executive dysfunction may be the primary neurocognitive mechanism for competency loss in PD.
Patients with PD demonstrated significant deficits across all four CCTI LS (LS1, LS3, LS4, LS5) (see table 1). As evidenced by increasing $\mathrm{F}$ ratios across these four LS (see table 1), the PD group demonstrated poorer performance on the LS as their difficulty increased. Similarly, competency outcomes of the patients with PD reflected a pattern of increasing compromise (marginally capable and incapable outcomes) across these four LS, ranging from a $30 \%$ compromise on LS1 to an $80 \%$ compromise on LS5 (see table 2). Similar to findings in studies with patients with $\mathrm{AD},{ }^{12}$ it appears that these four LS may be hierarchical in difficulty for patients with PD, with standards tapping reasoning (LS4) and information comprehension (LS5) being the most difficult and requiring a level of information retention and processing beyond the capacity of many patients with PD.

We were surprised that patients with PD showed impairments in performance and outcome on LS1, a minimal standard requiring only communication of a treatment choice (see tables 1 and 2). Examination of PD patient responses on LS1 indicated a tendency toward indecisiveness and tangentiality, and although most patients were able state a treatment choice, prompting was required in some cases. The CCTI score on LS1 is reduced if prompting is required; thus, there was a relatively high proportion of marginally competent outcomes for patients with PD on LS1 (25\%) (see table 2).

Patients with PD performed equivalently to controls on the ability to make the reasonable treatment choice (when the alternative is clearly unreasonable) 
([LS2]) (see table 1). Thus, cognitively impaired patients with PD appear to retain the ability to select a reasonable treatment option from one that is unreasonable.

Overall, the study findings raise significant concerns about the consent capacity of patients with PD. A large proportion of our patients with PD demonstrated impairments of consent capacity as measured by the CCTI. In particular, patients with PD had difficulty meeting more difficult, clinically relevant standards for competency, which tap reasoning and comprehension abilities. It is therefore likely that, in the usually protracted and progressive course of this illness, many patients with PD will become impaired, and in some cases, will become incapable of consenting to treatment. This conclusion highlights the importance of careful and continuing assessment of competency in patients with PD throughout the duration of their illness.

A second aim of the current study was to identify neurocognitive changes associated with declining competency in patients with PD. Our findings suggest that declines in simple executive function, and to a lesser extent memory, are key neurocognitive changes associated with competency loss in PD.

Executive dysfunction was closely associated with PD patient performance on LS5 (understanding treatment). Specifically, simple measures of executive function (EXIT 25) ${ }^{28}$ and memory (DRS Memory $)^{14}$ emerged as key predictors of LS5 performance, together accounting for $68 \%$ of LS5 score variance (see table 3). Using the EXIT 25, nonparametric DFA showed a very high classification rate of LS5 competency outcomes (90\%), which increased to $95 \%$ when both the EXIT 25 and DRS Memory were used (for additional information related to this article, please visit the Neurology Web site at www.neurology.org and scroll down the Table of Contents for the January 9 issue to find the title link for this article). The EXIT 25 is a bedside test of simple executive abilities, including verbal and spatial fluency, inhibition, primitive reflexes, and set flexibility. ${ }^{28}$ DRS Memory is a composite measure of memory that consists of short-term verbal recall items, orientation items, and verbal and visual recognition items. ${ }^{14,38}$ Thus, basic executive and to a lesser extent memory functions appear to mediate the capacity of patients with $\mathrm{PD}$ to comprehend a treatment situation and choices. This finding was consistent with the task demands of LS5, which require conceptual organization as well as recall of factually complex material. ${ }^{15}$

Executive dysfunction was also closely associated with PD performance on LS4 (rational reasons). Here again, the EXIT 25 was the key predictor of LS4 performance, accounting for $45 \%$ of LS4 score variance (see table 3). Using the EXIT 25, nonparametric DFA showed a very high classification rate of LS4 competency outcomes (90\%), which increased to
95\% when using both EXIT 25 and Trails B ${ }^{29}$ (for additional information related to this article, please visit the Neurology Web site at www.neurology.org and scroll down the Table of Contents for the January 9 issue to find the title link for this article). Trails B is a measure of visuomotor sequencing and set flexibility that is strongly associated with executive ability. ${ }^{29}$ Thus, basic executive functions also appear to mediate the capacity of patients with PD to provide rational reasons for a choice of medical treatment. This finding was consistent with the task demands of LS4, which require a subject to integrate information regarding two treatment choices and their risk/benefit profiles, and to provide logical reasons (pro and con) for his/her treatment choice.

Interestingly, simple memory and comprehension/ judgment abilities were associated with PD patient performance on LS1, a minimal standard requiring only communication of a treatment choice. DRS Memory emerged as the only multivariate predictor of the LS1 scores of patients with $\mathrm{PD}\left(\mathrm{R}^{2}=0.55, p=\right.$ 0.000 ) (see table 3 ), and it correctly classified $70 \%$ of patient competency outcomes (for additional information related to this article, please visit the Neurology Web site at www.neurology.org and scroll down the Table of Contents for the January 9 issue to find the title link for this article). When DRS Memory was coupled with WAIS-R Comprehension, they together correctly classified $100 \%$ of LS1 competency outcomes. These findings suggest that simple memory and also comprehension/judgment deficits underlie the declining capacity in patients with PD to communicate a treatment choice (LS1).

No neurocognitive variables emerged as predictors of LS3 or of [LS2]. Like controls, patients with PD showed almost perfectly intact performance on [LS2] (see table 1), which limited score variance and thus correlational analyses of the variable. In contrast, there was sufficient variance in LS3 scores, but no neuropsychological measure correlated significantly with LS3. Prior research with patients with AD has shown significant univariate and multivariate correlations between LS3 and several cognitive measures, particularly phonemic word fluency. ${ }^{23}$ It remains unclear why neurocognitive predictors failed to emerge for LS3 in the PD sample. One possibility is that the LS3 test items can draw strongly upon the subject's prior knowledge base, and may not always actually fully tap cognitive and emotional processes activated by the vignette. For example, one LS3 item asks: "How does your decision (not) to have brain surgery make you feel emotionally?" Such a question might be given a reasonable answer by patients with PD without any processing of vignette information on their part.

The neuropsychological findings are consistent with prior literature proposing a strong association between the frontal-subcortical disconnection syndrome of PD and executive dysfunction. ${ }^{4,7}$ The most 
problematic CCTI LS were the reasoning and comprehension standards (LS4 and LS5) that are cognitively most complex. ${ }^{12,15}$ Previous research has shown that patients with PD perform well on simple cognitive tasks, but as their complexity increases, performance deteriorates, which is likely a result of impaired executive control of higher order cognitive processes. $^{39,40}$ The findings are also consistent with research on patients with frontal lobe dementia and significant executive dysfunction who show impaired decision-making capacity despite intact language, memory, perception, and absence of apraxia and agnosia. ${ }^{41}$ Thus, the findings of the current study identify important relationships between cognitive impairment, executive dysfunction, and competency loss in PD.

The current study permits some preliminary comparisons to prior studies of consent capacity in $\mathrm{AD} .{ }^{12,23,42} \mathrm{As}$ in patients with $\mathrm{AD}$, our $\mathrm{PD}$ group showed impaired consent capacity on LS3 to LS5, with a hierarchical pattern on these standards associated with dementia level and difficulty of standard. Also like patients with $\mathrm{AD}$, our $\mathrm{PD}$ group performed equivalently with controls on [LS2], which requires distinguishing the reasonable treatment option from a clearly unreasonable alternative. However, although patients with $\mathrm{AD}$ generally have not differed from controls on $\mathrm{LS} 1,{ }^{12}$ patients with PD in the current study did show impairment on this minimal standard (see table 1), which may relate to tangentiality secondary to executive dysfunction. On more stringent standards involving complex information processing and reasoning (LS3, LS4, and LS5), the PD group appears to perform better than both mild and moderate $\mathrm{AD}$ groups. ${ }^{12}$ Although additional empirical research is needed here, such a finding would not be surprising, as neuropsychological research comparing $\mathrm{AD}$ and $\mathrm{PD}$ groups has shown significantly better memory and reasoning skills in patients with $\mathrm{PD}$ than in those with $\mathrm{AD} .{ }^{43,44} \mathrm{Fi}$ nally, the CCTI competency predictor profiles of the $\mathrm{PD}$ and $\mathrm{AD}$ groups seem to differ insofar as executive dysfunction is the preeminent predictor of competency for the PD group, whereas a variety of cognitive functions have predicted CCTI competency in patients with $\mathrm{AD} .{ }^{23,42}$ This observed difference in predictor sets also requires more systematic investigation, but quite possibly reflects the subcortical pathology and more limited deficit profile of $\mathrm{PD}$, versus the widespread cortical pathology and global cognitive impairment characteristic of $\mathrm{AD}$.

Some limitations of the current study should be noted. First, the relatively small samples of controls and patients with PD suggest some caution concerning the generalizability of the results. Second, the psychometrically based performance and outcome results of the CCTI should not be construed as an actual index of a subject's legal competency. Rather, the CCTI is an adjunct objective tool to be used by health-care professionals, in conjunction with other clinical and historical information, to make clinical judgments about the quality of an individual's medical decision making. Although the current study indicated deficits in the performance and outcomes of patients with PD on the CCTI, such findings must not be confused with legal incompetency, which is a judgment of the legal system. Finally, the vignette methodology employed in the current study has certain limitations. Decision making concerning a real and personal medical problem is arguably distinct from decision making in a hypothetical medical situation. ${ }^{12,16}$ It is possible that patients facing real, personal medical issues might demonstrate treatment decision-making approaches somewhat differently than those elicited by the CCTI.

\section{Acknowledgment}

The authors thank Susan Kotler, PhD, Mark Mennemeier, PhD, and Kelly Earnst, $\mathrm{PhD}$, for comments regarding the study.

\section{References}

1. Mayeux R, Denaro J, Hemenegildo N, et al. A population based investigation of Parkinson's disease with and without dementia. Relationship to age and gender. Arch Neurol 1992; 49:492-497.

2. Aarsland D, Tandberg E, Larsen J, Cummings J. Frequency of dementia in Parkinson's disease. Arch Neurol 1996;53:538542.

3. Hughes T, Ross H, Musa S, et al. A 10-year study of the incidence of and factors predicting dementia in Parkinson's disease. Neurology 2000;54:1596-1602.

4. Mahurin R, Feher E, Nance M, Levy J, Pirozzolo F. Cognition in Parkinson's disease and related disorders. In: Parks R, Zec R, Wilson R, eds. Neuropsychology of Alzheimer's disease and other dementias. New York, NY: Oxford University Press, 1993:247-268.

5. Brown R, Marsden C. Internal versus external cues in the control of attention in Parkinson's disease. Brain 1988;111: $323-345$.

6. Levin B, Tomer R, Rey G. Clinical correlates of cognitive impairment in Parkinson's disease. In: Huber S, Cummings J, eds. Parkinson's disease: neurobehavioral aspects. New York, NY: Oxford University Press, 1992:97-106.

7. White R, Au R, Durso R, Moss M. Neuropsychological function in Parkinson's disease. In: White R, ed. Clinical syndromes in adults: the practitioner's handbook. New York, NY: Elsevier Science, 1992:253-286.

8. Grisso T. Evaluating competencies: forensic assessments and instruments. New York, NY: Plenum Press, 1986.

9. Meisel A, Roth L, Lidz C. Toward a model of the legal doctrine of informed consent. Am J Psychiatry 1977;134:285-289.

10. Kapp M. Geriatrics and the law: patient rights and professional responsibilities. New York, NY: Springer, 1992.

11. Merz J. On a decision-making paradigm of medical informed consent. J Leg Med 1993;14:231-264.

12. Marson DC, Ingram KK, Cody HA, Harrell LE. Assessing the competency of patients with Alzheimer's disease under different legal standards. Arch Neurol 1995;52:949-954.

13. Marson DC, McInturff B, Hawkins L, Bartolucci A, Harrell LE. Consistency of physician judgments of capacity to consent in mild Alzheimer's disease. J Am Geriatr Soc 1997;45:453457.

14. Mattis S. Dementia rating scale. In: Bellack R, Karasu B, eds. Geriatric psychiatry. New York, NY: Grune \& Stratton, 1976: 77-119. 
15. Dymek MP, Marson DC, Harrell L. Factor structure of capacity to consent to medical treatment in patients with Alzheimer's disease: an exploratory study. J Forensic Neuropsychol 1999;1:27-48.

16. Marson D, Annis S, McInturff B, Bartolucci A, Harrell L. Error behaviors associated with loss of competency in Alzheimer's disease. Neurology 1999;53:1983-1992.

17. Roth L, Meisel A, Lidz C. Tests of competency to consent to treatment. Am J Psychiatry 1977;134:279-284.

18. Appelbaum P, Grisso T. Assessing patients' capacities to consent to treatment. N Engl J Med 1988;319:1635-1638.

19. Tepper A, Elwork A. Competency to consent to treatment as a psychological construct. Law Hum Behav 1984;8:205-223.

20. Marson D, Earnst K, Jamil F, Bartolucci A, Harrell L. Consistency of physicians' legal standard and personal judgments of competency in patients with Alzheimer's disease. J Am Geriatr Soc 2000;48:911-918.

21. Grisso T, Appelbaum P. Mentally ill and non-mentally ill patients' abilities to understand informed consent disclosure for medication. Law Hum Behav 1991;15:377-388.

22. Alexander M. Clinical determination of mental competence. Arch Neurol 1988;45:23-26.

23. Marson DC, Chatterjee A, Ingram KK, Harrell LE. Toward a neurologic model of competency: cognitive predictors of capacity to consent in Alzheimer's disease using three different legal standards. Neurology 1996;46:666-672.

24. Wechsler D. Wechsler Memory Scale-Revised. New York, NY: The Psychological Corporation, 1987.

25. Kaplan E, Goodglass H, Weintraub S. Boston Naming Test. Philadelphia, PA: Lea \& Febiger, 1983.

26. Eisenson J. Examining for aphasia. New York, NY: The Psychological Corporation, 1954.

27. Spellacy F, Spreen O. A short form of the Token Test. Cortex 1969;5:390-397.

28. Royall D, Mahurin R, Gray K. Bedside assessment of executive cognitive impairment: the Executive Interview. J Am Geriatr Soc 1992;40:1221-1226.

29. Reitan R. Validity of the Trail Making Test as an indication of organic brain damage. Percept Mot Skills 1958;8:271-276.

30. Benton A, Hamsher K. Multilingual aphasia examination. Iowa City, IA: The University of Iowa, 1978.
31. Lezak M. Neuropsychological assessment, 3rd ed. New York, NY: Oxford University Press, 1995.

32. Wechsler D. Wechsler Adult Intelligence Scale-Revised. New York, NY: The Psychological Corporation 1981.

33. Yesavage J. Development and validation of a geriatric depression screening scale: a preliminary report. J Psychiatr Res 1983;17:37-49.

34. Norusis M. SPSS advanced statistics 6.1. Chicago, IL: SPSS Inc, 1994.

35. Tabachnick BG, Fidell LS. Using multivariate statistics. New York, NY: Harper Collins, 1996.

36. SAS Institute I. User's guide, version 5. Cary, NC: SAS Institute Inc., 1985.

37. Marson DC, Harrell LE. Neurocognitive models that predict physician judgments of capacity to consent in patients with mild Alzheimer's disease. In: Park D, Morrell R, Shifrin K, eds. Medical information processing and aging. New York, NY: Lawrence Erlbaum, 1999:109-126.

38. Marson DC, Dymek MP, Duke LW, Harrell LE. Subscale validity of the Mattis Dementia Rating Scale. Arch Clin Neuropsychol 1996;12:269-275.

39. Gotham A, Brown R, Marsden C. "Frontal" cognitive functions in patients with Parkinson's disease "on" and "off" levodopa. Brain 1988;111:199-231.

40. Girotti G, Grassi M, Carella F, et al. Possible involvement in attention processes in Parkinson's disease. In: Yahr M, Bergmann K, eds. Advances in neurology. New York, NY: Raven Press, 1986:425-429.

41. Schindler B, Rachmandi D, Matthews M, Podell K. Competency and the frontal lobe: the impact of executive dysfunction on decisional capacity. Psychosomatics 1995;36:400-404.

42. Marson DC, Cody HA, Ingram KK, Harrell LE. Neuropsychologic predictors of competency in Alzheimer's disease using a rational reasons legal standard. Arch Neurol 1995;52:955-959.

43. Livitan I, Moore E, Williams J, Gomez C, Chase T. Differential memory and executive functions in demented patients with Parkinson's and Alzheimer's disease. J Neurol Neurosurg Psychiatry 1991;54:25-39.

44. Paolo A, Troster A, Glatt S, Hubble J, Koller W. Differentiation of the dementias of Alzheimer's and Parkinson's disease with the Dementia Rating Scale. J Geriatr Psychiatr Neurol 1995;8:184-188. 


\section{Neurology}

\section{Competency to consent to medical treatment in cognitively impaired patients with Parkinson's disease}

Maureen P. Dymek, Paul Atchison, Lindy Harrell, et al. Neurology 2001;56;17-24

DOI 10.1212/WNL.56.1.17

\section{This information is current as of January 9, 2000}

\section{Updated Information \&} Services

Supplementary Material

\section{References}

Permissions \& Licensing

Reprints including high resolution figures, can be found at: http://n.neurology.org/content/56/1/17.full

Supplementary material can be found at: http://n.neurology.org/content/suppl/2000/12/11/56.1.17.DC1

This article cites 27 articles, 4 of which you can access for free at: http://n.neurology.org/content/56/1/17.full\#ref-list-1

Information about reproducing this article in parts (figures,tables) or in its entirety can be found online at:

http://www.neurology.org/about/about_the_journal\#permissions

Information about ordering reprints can be found online:

http://n.neurology.org/subscribers/advertise

Neurology ${ }^{\circledR}$ is the official journal of the American Academy of Neurology. Published continuously since 1951, it is now a weekly with 48 issues per year. Copyright . All rights reserved. Print ISSN: 0028-3878. Online ISSN: 1526-632X.

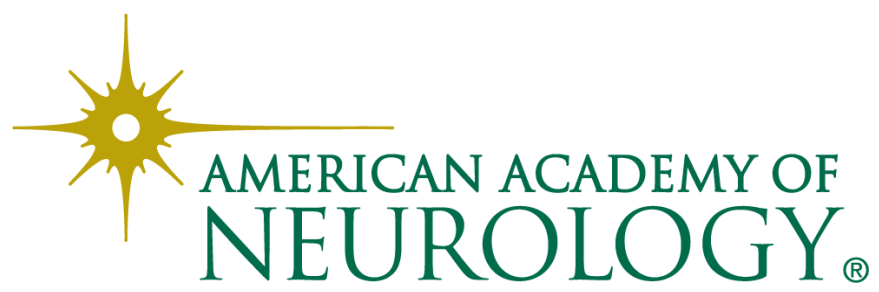

